\title{
Experiments with ultracold neutrons
}

\author{
V.V. Nesvizhevsky \\ Institut Laue-Langevin, 6 rue Jules Horowitz, Grenoble F-38046, France \\ E-mail: nesvizh@ill.fr
}

Received December 12, 2010

\begin{abstract}
Ultracold neutrons (UCN) form a tiny low-energy fraction in Maxwelian spectrum of thermal neutrons in moderators of nuclear reactors and spallation sources. Their energy is extremely small $\left(\sim 10^{-7} \mathrm{eV}\right)$, their velocity is a few meters per second, and their effective temperature is as low as $\sim 1 \mathrm{mK}$. Specific feature of UCN consists of their nearly total elastic reflection from nuclear-optical potential of many materials at any incidence angle; therefore they could be stored in closed traps for many minutes, thus they could be used for extremely sensitive measurements. A fraction of UCN in the thermal neutron flux is as low as $10^{-11}-10^{-12}$, and serious efforts are undertaken all over the world to produce $\mathrm{UCN}$ in larger amounts. UCN are widely used in precision particle physics experiments. Applications of UCN are emerging in surface and nanoparticle physics. Here we will focus on recent advances in the field.
\end{abstract}

PACS: 14.20.Dh Protons and neutrons;

03.75.Be Atom and neutron optics;

29.25.Dz Neutron sources.

Keywords: ultracold neutrons, fundamental physics, quantum phenomena.

\section{Introduction}

The present contribution is based on a talk given at the School of Young Scientists carried out during 8th Conference on Cryocrystals and Quantum Crystals in Chernogolovka, Russia, on 26-31 July 2010. This contribution presents the physics with ultracold neutrons; particular attention is paid to recent advances related to the domain of interest of the author of present contribution.

Ultracold neutrons (UCN) [1-4] form a tiny low-energy fraction in Maxwelian spectrum of thermal neutrons in moderators of nuclear reactors and spallation sources. Their energy is extremely small $\left(\sim 10^{-7} \mathrm{eV}\right)$, their velocity equals a few meters per second only, and their effective temperature is as low as $\sim 1 \mathrm{mK}$. Specific feature of UCN consists of their nearly total elastic reflection from the nuclear-optical potential of many materials at any incidence angle; therefore they could be stored in closed traps for extended period of time, thus they could be used for extremely sensitive measurements. UCN characteristic penetration depth is close to its wavelength and equal to a few tens nanometers. Very cold neutrons (VCN) with typical energy of $10^{-7}-10^{-4} \mathrm{eV}$ are totally reflected from flat surface only if the incidence angle is sufficiently small; so that the neutron longitudinal velocity component is lower than the material critical velocity. As a fraction of UCN in the thermal neutron flux is as low as $10^{-11}-10^{-12}$, serious effort are undertaken all over the world to produce UCN in larger amounts, using super-thermal UCN sources or even equilibrium cooling of neutrons [5-20]. UCN are widely used in precision particle physics experiments [21], such as, for instance, searches for additional fundamental shortrange forces [22-28], searches for non-zero neutron electric dipole moment [29,30], precision neutron lifetime measurements [31-36] and constrains for the neutron electric charge [37,38]. Applications of UCN are emerging in surface and nanoparticle physics $[39,40]$. We focus on recent advances in the field including observation of the centrifugal quantum states of neutrons. Combined with observation of the gravitationally bound quantum states of neutrons, this phenomenon provides the first demonstration of the weak equivalence principle for an object in a quantum state $[41,42]$. Also we will present a new spectrometer GRANIT constructed for precision studies of the gravitationally bound quantum states of neutrons and for other applications in particle physics, quantum optics, and in surface studies [43]. A promising methodical development in the field consists of building neutron reflectors based on nanostructured materials [44-46]. Finally, unique properties of UCN allow using them for experimental studies of motions of weakly bound nanoparticles $[13,47]$. 


\section{Centrifugal and gravitational quantum states of neutrons}

Lift a ping-pong ball to a height $H_{0}$ above table and let it gently falling down. The ball will accelerate in the Earth's gravity field to the velocity $V_{0}=\sqrt{2 g H_{0}}, g$ is the gravitational acceleration; then it will reflect from the table surface. In case of perfectly elastic reflection in vacuum, the ball would return back to surface due to gravity after the period of $\Delta \tau_{0}\left(H_{0}\right)=\sqrt{8 H_{0} / g}$; then it would continue bouncing with the frequency $v_{0}\left(H_{0}\right)=1 / \tau_{0}\left(H_{0}\right)=$ $=\sqrt{\mathrm{g} / 8 H_{0}}$. The smaller $H_{0}$, the larger $v_{0}: v_{0}(1 \mathrm{~m}) \approx$ $\approx 10^{0} \mathrm{~Hz}, v_{0}(1 \mathrm{~cm}) \approx 10^{1} \mathrm{~Hz}, v_{0}(100 \mu \mathrm{m}) \approx 10^{2} \mathrm{~Hz}$. The frequency $v_{0}$ does not depend on the ball mass $M$.

Imagine another experiment (Fig. 1). A table is moving together with a spaceship with the acceleration $\mathbf{a}=-\mathbf{g}$ far from large gravitating masses; the table surface is perpendicular to g. An observer in the spaceship will see a ball bouncing on the table with the same frequency $v_{0}$ as it does in the previous experiment (an observer in the rest frame will see the table accelerating towards the ball). This is a consequence of the weak equivalent principle verified with amazing accuracy of $\sim 10^{-12}$ for macroscopic objects [48], and with the accuracy of $\sim 3 \cdot 10^{-4}$ for a classical elementary particle [49].

What would happen in the two experiments at very small heights $H_{0}$ ? Would be these two problems still equivalent? Would the frequency seek to infinity? No, the frequency would increase only if $H_{0}$ exceeds the quantum-mechanical limit $H_{0}^{Q M}$ that could be estimated using Heisenberg coordinate-momentum uncertainty relation: $H_{0}^{Q M} M \sqrt{2 g H_{0}^{Q M}} \approx 2 \pi \hbar$, where $\hbar$ is the Planck constant. For a ball in the Earth's gravitational field the value $H_{0}^{Q M}$ is too small. However, quantum effects for an elementary particle, for instance, for a neutron, could be observed at

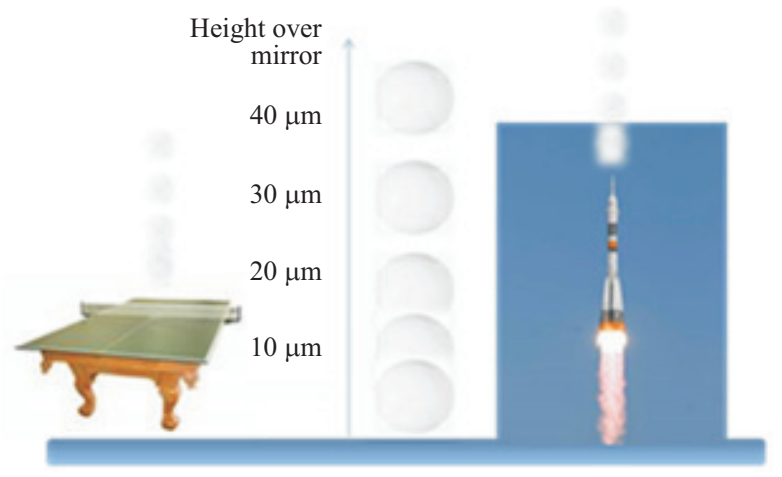

Fig. 1. The quantum behavior of an object above mirror in the gravity field and that in the accelerating reference system is illustrated schematically. The ball heights correspond to its most probable positions; the scale corresponds to the neutron mass, we consider 5 th quantum state. relatively large height of $H_{0}^{Q M} \approx 10 \mu m$ [41,50,51]. The condition separating quantum and classical behavior of UCN above mirror is defined by a ratio of a neutron quantum state width $\delta E_{n}$ (reciprocal neutron lifetime $\tau_{n}^{-1}$ in $n$th quantum state) and an energy difference between neighbor quantum states $\Delta E_{n+1, n}=E_{n+1}-E_{n}$ (i.e. the energy-time uncertainty relation $\left.\tau_{n} \Delta E_{n, n+1} \approx 2 \pi \hbar\right)$. The transition from a classical case to a quantum one is considered, for instance, in Refs. 52,53.

In a quantum limit, we do not consider trajectories, heights, velocities; a frequency is defined by an energy as $v_{0}^{Q M} \approx E_{0} /(2 \pi \hbar)$, and a characteristic height $H_{0}^{Q M}$ depends on mass. In accordance with the weak equivalence principle, an effective centrifugal potential [42,54] is locally equivalent to gravity. Thus objects do not fall in gravitational field and they do not move in an accelerated reference system universally: massive objects could behave classically while light objects exhibit quantum properties at equal distances to mirror. Nevertheless, the weak equivalence principle holds: it means in our case that neutron quantum states in gravitational and centrifugal potentials are equivalent if accelerations are equal.

A general solution of Schrödinger equation for a particle above mirror attracted by linear potential was found in 1920th [55]. Nevertheless, it was regarded for a long time just as a beautiful quantum-mechanical text-book problem [56-62]. However, conditions corresponding to this idealized problem have been realized recently in experiments with slow neutrons in gravitational [41] and centrifugal [42] potentials in Institut Laue-Langevin, Grenoble, France. Due to limited space, we do not present these experiments in detail here, but refer our readers to detailed overviews $[63,64]$, or/and to many other relevant publications [50,51,65-78].

\section{GRANIT spectrometer}

Further, more precise studies of gravitational and centrifugal quantum states of neutrons will continue in an advanced GRANIT spectrometer that is currently under commissioning in Institut Laue-Langevin in Grenoble, France $[43,79]$. GRANIT is a follow-up project based on a secondgeneration UCN gravitational spectrometer with ultra-high energy resolution. The studies will focus on applications of these phenomena in fundamental particle physics, surface studies, methodical applications, and reflectometry with $\mathrm{UCN}$ as well as in quantum optics.

Basic advantages of the new GRANIT spectrometer include 1) much longer observation time of neutrons in a closed specular trap [72,80-82] thus much better precision in energy measurements; 2) the method of resonance transitions between the gravitationally bound quantum states of neutrons [72,79]; 3) increase in UCN density using dedicated ${ }^{4} \mathrm{He}$ UCN source [20] delivering UCN to the spectrometer with no significant dilution of phase-space density 
$[83,84]$; 4) profiting from a permanent installation of the spectrometer in a more comfortable experimental environment; 5) using polarized neutrons and polarization analysis.

\section{Nanoparticle reflectors for slow neutrons and studies of weakly bound nanoparticles}

Significant advance in using nano-structures in UCN physics on one hand and in studying nanoparticles and nano-structures using $\mathrm{UCN}$ on the other hand is due to fortunate coincidence of some their characteristic parameters [13]. Thus, under certain conditions, the neutron wavelength is close to nanoparticles size, simultaneously the neutron velocity is about equal to the thermal velocity of nanoparticles.

Recently, powders of diamond nanoparticles have been used efficiently as the first VCN reflectors in the complete velocity range from $\mathrm{UCN}$ to up to $\sim 160 \mathrm{~m} / \mathrm{s}$, thus bridging the energy gap between efficient reactor reflectors for thermal and cold neutrons, and optical neutron-matter potential for UCN (see Fig. 2) [13,44,85]. Moreover, VCN could be stored in traps with nano-structured walls in some analogy to storage of UCN in traps [45]. Diamond, with its exceptionally high optical nuclear potential and low absorption cross-section, is a particularly suitable material for this application. Formation of diamond nanoparticles by explosive shock was first observed 50 years ago [86]. These particles measure a few nanometers. They consist of a diamond nucleus (with a typical diamond density and optical nuclear potential) within an onion-like shell of a complex chemical composition [87] (with significantly

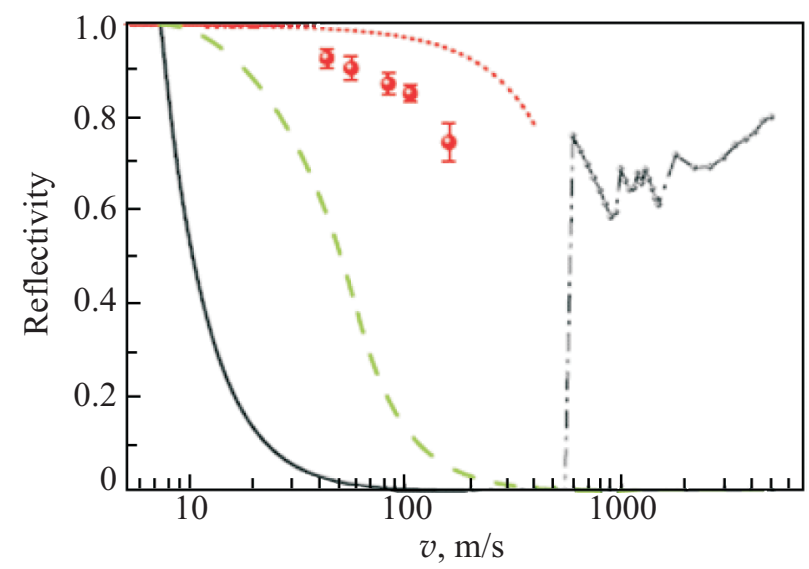

Fig. 2. The elastic reflection probability for isotropic neutron flux is shown as a function of the neutron velocity for various carbonbased reflectors: (1) Diamond-like coating (DLC) (thin solid line), (2) The best supermirror (dashed line), (3) Hydrogen-free ultradiamond powder with the infinite thickness (dotted line). Calculation. (4) VCN reflection from $3 \mathrm{~cm}$-thick diamond nanopowder at ambient temperature (points), with significant hydrogen contamination. Experiment. (5) MCNP calculation for reactor graphite reflector with the infinite thickness at ambient temperature (dashed-dotted line). lower optical potential). The use of nanoparticles with the characteristic size of a few nanometers is needed to provide a sufficiently large cross-section of coherent interaction and inhomogeneity of the reflector density on a spatial scale of about the neutron wavelength. A large number of diffusive large-angle neutron-nanoparticle scattering events needed to reflect $\mathrm{VCN}$ from powder constrains the choice of materials: only low absorbing materials with high optical potential are appropriate.

Studying so-called anomalous losses [88] of UCN from traps (providing an obstacle for precision neutron lifetime experiments) we observed a surprising phenomenon: the energy of stored UCN increased by $\sim 10^{-7} \mathrm{eV}$ with the probability of $\sim 10^{-8}-10^{-5}$ per collision [89]; this value exceeded any theoretical expectations by many orders of magnitude. If the neutron energy after such inelastic scattering exceeds some critical value it would escape from the trap. This small heating of UCN has been studied over the last years both on solid surfaces (stainless steel, copper, beryllium etc) and on liquid surfaces (different kinds of hydrogen-free oils) [47,89-98]. Only the scattering of UCN at weakly bound nanoparticles on surface with a size of $\sim 10 \mu \mathrm{m}$ can explain the experimental data obtained [13]. To our knowledge, such quasi-elastic scattering of UCN provides unique opportunity to measure slow motions of nano-objects as well as to study their interaction with surfaces and with each other. Impurity gels [99-105] provide an interesting object to study using neutron techniques, as well as a tool to reflect and even to slow down neutrons $[13,106,107]$ using the observed earlier quasi-elastic reflection of slow neutrons.

\section{Summary}

We presented recent experiments with UCN and discussed further prospects in the field. These studies as well as many other applications of slow neutrons are rapidly progressing.

The author is sincerely grateful to all colleagues contributed to the studies overviewed here, in particular to GRANIT collaborators. These experiments are supported in part by GRANIT collaboration, by ANR (Agence Nationale de la Recherche, France), and the Federal program "Scientific and pedagogical cadres of innovative Russia".

1. V.I. Luschikov, Y.N. Pokotilovsky, A.V. Strelkov, and V.F. Shapiro, JETP Lett. 9, 23 (1969).

2. V.K. Ignatovich, The Physics of Ultracold Neutrons, Clarendon Press, Oxford (1990).

3. R. Golub, D.J. Richardson, and S.K. Lamoreux, Ultracold Neutrons, Higler, Bristol (1991).

4. A.V. Strelkov, Usp. Fiz. Nauk 174, 565 (2004).

5. R. Golub and J.M. Pendlebury, Phys. Lett. A53, 133 (1975).

6. A. Steyerl, H. Nagel, F.X. Schreiber, K.A. Steinhauser, R. Gähler, W. Gläser, P. Ageron, J.M. Astruc, W. Drexel, G. Gervais, and W. Mampe, Phys. Lett. A116, 347 (1986). 
7. I.S. Altarev, N.V. Borovikova, A.P. Bulkin, V.V. Vesna, E.A. Garusov, L.A. Grigorieva, A.I. Egorov, B.G. Erozolimski, A.N. Erykalov, A.A. Zakharov, S.N. Ivanov, V.Ya. Kezerashvili, S.G. Kirsanov, E.A. Kolomenski, K.A. Konoplev, I.A. Kuznetsov, V.M. Lobashev, N.F. Maslov, V.A. Mitykhlyaev, I.S. Okunev, B.G. Peskov, Yu.V. Petrov, P.G. Pikulik, N.A. Pirozhkov, G.D. Porsev, A.P. Serebrov, Yu.V. Sobolev, R.R. Taldaev, V.A. Shustov, and A.F. Shchebetov, JETP Lett. 44, 344 (1986).

8. A.P. Serebrov, V.A. Mityukhlyaev, A.A. Zakharov, V.V. Nesvizhevsky, and A.G. Kharitonov, JETP Lett. 59, 757 (1994).

9. A.P. Serebrov, V.A. Mityukhlyaev, A.A. Zakharov, A.G. Kharitonov, V.V. Nesvizhevsky, M.S. Lasakov, R.R. Tal'daev, A.V. Alduschenkov, V.E. Varlamov, and A.V. Vasyliev, JETP Lett. 62, 785 (1995).

10. Yu.N. Pokotilovski, Nucl. Instrum. Methods A356, 412 (1995).

11. B.V. Bagrjanov, D.G. Kartashov, M.I. Kuvshinov, A.Yu. Muzychka, G.V. Nekhaev, A.D. Rogov, I.G. Smirnov, A.D. Stoica, A.V. Strelkov, and V.N. Shvetsov, Phys. At. Nucl. 62, 787 (1999).

12. U. Trinks, F.G. Hartmann, S. Paul, and W. Schott, Nucl. Instrum. Methods A440, 666 (2000).

13. V.V. Nesvizhevsky, Phys. At. Nucl. 65, 400 (2002).

14. Y. Masuda, T. Kitagaki, K. Hatanaka, M. Higuchi, S. Ishimoto, Y. Kiyanagi, K. Morimoto, S. Muto, and M. Yoshimura, Phys. Rev. Lett. 89, 284801 (2002).

15. C.A. Baker, S.N. Balashov, J. Butterworth, P. Geltenbort, K. Green, P.G. Harris, van der M.G. GritenD., P.S. Iaydjiev, S.N. Ivanov, J.M. Pendlebury, D.B. Schiers, M.A.H. Tucker, and H. Yoshiki, Phys. Lett. A308, 67 (2003).

16. A. Saunders, J.M. Anaya, T.J. Bowles, B.W. Filippone, P. Geltenbort, R.E. Hill, M. Hino, S. Hoedl, G.E. Hogan, T.M. Ito, K.W. Jones, T. Kawai, K. Kirch, S.K. Lamoreaux, C.-Y. Liu, M. Makela, L.J. Marek, J.W. Martin, C.L. Morris, R.N. Mortensen, A. Pichlmaier, S.J. Seestrom, A. Serebrov, D. Smith, W. Teasdale, B. Tipton, R.B. Vogelaar, A.R. Young, and J. Yuan, Phys. Lett. B593, 55 (2004).

17. F. Atchison, van der B. Brandt, T. Brys, M. Daum, P. Fierlinger, P. Hautle, R. Henneck, S. Heule, M. Karprzak, K. Kirch, J.A. Konter, A. Michels, A. Pichlmaier, M. Wohlmuther, and A. Wokaun, Phys. Rev. C71, 054601 (2005).

18. O. Zimmer, K. Baumann, M. Fertl, B. Franke, S. Mironov, C. Plonka, D. Rich, P. Schmidt-Wellenburg, H.-F. Wirth, and B. van der Brandt, Phys. Rev. Lett. 99, 104801 (2007).

19. D. Bondoux, H.G. Börner, V. Ermilov, J.P. Gonzales, E. Kulagin, S. Kulikov, E. Lelievre-Berna, V. Melikhov, V.V. Nesvizhevsky, T. Soldner, F. Thomas, and E. Shabalin, Nucl. Instrum. Methods A606, 637 (2009).

20. P. Schmidt-Wellenburg, K.H. Andersen, P. Courtois, M. Kreuz, S. Mironov, V.V. Nesvizhevsky, G. Pignol, K.V. Protasov, T. Soldner, F. Vezzu, and O. Zimmer, Nucl. Instrum. Methods A611, 267 (2009).

21. V. Nesvizhevsky, C. Plonka-Spehr, K. Protasov, K. Schreckenbach, T. Soldner, and O. Zimmer, Nucl. Instrum. Methods A611, 1 (2009).
22. O. Bertolami and F.M. Nunes, Class. Quant. Grav. 20, L61 (2003).

23. H. Abele, S. Bäßler, and A. Westphal, Lecture Notes in Physics 631, 355 (2003).

24. V.V. Nesvizhevsky and K.V. Protasov, Class. Quant. Grav. 21, 45576 (2004).

25. S. Bäßler, V.V. Nesvizhevsky, K.V. Protasov, and A.Yu. Voronin, Phys. Rev. D75, 075006 (2007).

26. V.V. Nesvizhevsky, G. Pignol, and K.V. Protasov, Phys. Rev. D77, 034020 (2008).

27. A.P. Serebrov, O. Zimmer, P. Geltenbort, A.K. Fomin, S.N. Ivanov, E.A. Kolomensky, I.A. Krasnoshchekova, M.S. Lasakov, V.M. Lobashev, A.N. Pirozhkov, V.E. Varlamov, A.V. Vasiliev, O.M. Zherebtsov, E.B. Aleksandrov, S.P. Dmitriev, and N.A. Dovator, JETP Lett. 91(1), 6 (2010).

28. S. Bäßler, V.V. Nesvizhevsky, G. Pignol, K.V. Protasov, and A.Yu. Voronin, Nucl. Instrum. Methods A611, 149 (2009).

29. I.S. Altarev, Yu.N. Borisov, N.V. Borovikova, S.N. Ivanov, E.A. Kolomensky, M.S. Lasakov, V.M. Lobashev, V.A. Nazarenko, A.N. Pirozhkov, and A.P. Serebrov, Phys. Lett. B276, 242 (1992).

30. C.A. Baker, D.D. Doyle, P. Geltenbort, K. Green, van der M.G.D. Grinten, P.G. Harrison, P. Iaydjiev, S.N. Ivanov, D.G. Mayr, J.M. Pendlebury, D. Richardson, D. Shiers, and K.F. Smith, Phys. Rev. Lett. 97, 131801 (2006).

31. W. Mampe, P. Ageron, C. Bates, J.M. Pendlebury, and A. Steyerl, Phys. Rev. Lett. 63, 593 (1989).

32. V.V. Nesvizhevsky, A.P. Serebrov, R.R. Tal'daev, A.G. Kharitonov, V.P. Alfimenkov, A.V. Strelkov, and V.N. Shvetsov, JETP 75, 405 (1992).

33. W. Mampe, L.N. Bondarenko, V.I. Morozov, Yu.N. Panin, and A.I. Fomin, JETP Lett. 57, 82 (1993).

34. S. Arzumanov, L. Bondarenko, S. Chernyavsky, W. Drexel, A. Fomin, P. Geltenbort, V. Morozov, Yu. Panin, M. Pendlebury, and K. Schreckenbach, Phys. Lett. B483, 15 (2000).

35. A. Pichlmaier, J. Butterworth, P. Geltenbort, H. Nagel, V.V. Nesvizhevsky, S. Neumaier, K. Schreckenbach, S. Steichele, and V. Varlamov, Nucl. Instrum. Methods A440, 517 (2000).

36. A.P. Serebrov, V.E. Varlamov, A.G. Kharitonov, A.K. Fomin, Yu.N. Pokotilovski, P. Geltenbort, I.A. Krasnoschekova, M.S. Lasakov, R.R. Tal'daev, and A.S. Vassiljev, Phys. Rev. C78, 035505 (2008).

37. Yu.V. Borisov, N.V. Borovikova, A.V. Vasilyev, L.A. Grigorieva, S.N. Ivanov, N.T. Kashukeev, V.V. Nesvizhevsky, A.P. Serebrov, and P.S. Iadjiev, J. Techn. Phys. 58, 951 (1988).

38. C. Plonka-Spehr, P. Geltenbort, P. Iaydjiev, J. Klepp, V.V. Nesvizhevsky, T. Lauer, and A. Kraft, Nucl. Instrum. Methods A., Vol. in press (2010).

39. L. Bondarenko, S. Chernyavsky, A. Fomin, P. Geltenbort, V. Morozov, and S. Shilkin, Physica B234, 11891 (1997).

40. E.V. Lychagin, A.Yu. Muzychka, V.V. Nesvizhevsky, G.V. Nekhaev, G. Pignol, K.V. Protasov, and A.V. Strelkov, Nucl. Instrum. Methods A611, 302 (2009).

41. V.V. Nesvizhevsky, H.G. Börner, A.K. Petoukhov, H. Abele, S. Bäßler, F.J. Rueß, Stöferle, Th., A. Westphal, A.M. Gagarski, G.A. Petrov, and A.V. Strelkov, Nature 415, 297 (2002). 
42. V.V. Nesvizhevsky, A.Yu. Voronin, R. Cubitt, and K.V. Protasov, Nature Phys. 6, 114 (2010).

43. http://lpsc.in2p3.fr/Indico/conferenceDisplay.py? confId $=371$. [Online]

44. V.V. Nesvizhevsky, E.V. Lychagin, A.Yu. Muzychka, A.V. Strelkov, G. Pignol, and K.V. Protasov, Nucl. Instrum. Methods A595, 631 (2008).

45. E.V. Lychagin, A.Yu. Muzychka, V.V. Nesvizhevsky, G. Pignol, K.V. Protasov, and A.V. Strelkov, Phys. Lett. B679, 186 (2009).

46. V. Nesvizhevsky, R. Cubitt, E. Lychagin, A. Muzychka, G. Nekhaev, G. Pignol, K. Protasov, and A. Strelkov, Materials 3, 1768 (2010).

47. D.G. Kartashov, E.V. Lychagin, A.Yu. Muzychka, V.V. Nesvizhevsky, G.V. Nekhaev, and A.V. Strelkov, Intern. J. Nanoscience 6, 501 (2007).

48. Y. Su, B.R. Heckel, E.G. Adelberger, J.H. Gundlach, M. Harris, G.L. Smith, and H.E. Swanson, Phys. Rev. D50, 3614 (1994).

49. L. Koester, Phys. Rev. D14, 907 (1976).

50. V.V. Nesvizhevsky, H.G. Börner, A.M. Gagarski, A.K. Petoukhov, G.A. Petrov, H. Abele, S. Bäßler, G. Divkovic, F.J. Rueß, Stöferle, Th., A. Westphal, A.V. Strelkov, K.V. Protasov, and A.Yu. Voronin, Phys. Rev. D67, 102002 (2003).

51. V.V. Nesvizhevsky, A.K. Petoukhov, H.G. Börner, T.A. Baranova, A.M. Gagarski, G.A. Petrov, K.V. Protasov, A.Yu. Voronin, S. Bäßler, H. Abele, A. Westphal, and L. Lucovac, Europ. Phys. J. C40, 479 (2005).

52. O. Bertolami and J.G. Rosa, Phys. Lett. B633, 111 (2006).

53. M. Berberan-Santos, E. Bodunov, and L. Pogliani, J. Math. Chem. 37, 101 (2005).

54. V.V. Nesvizhevsky, A.K. Petukhov, K.V. Protasov, and A.Yu. Voronin, Phys. Rev. A78, 033616 (2008).

55. G. Breit, Phys. Rev. 32, 273 (1928).

56. I.I. Gol'dman, V.D. Krivchenkov, V.I. Kogan, and V.M. Galitskii, Problems in Quantum Mechanics, Academic, New York (1960).

57. D. ter Haar, Selected Problems in Quantum Mechanics, New York, Academic Press (1964).

58. L. Landau and E. Lifshits, Quantum Mechanics. Nonrelativistic Theory, Pegamon, London (1965).

59. P.W. Langhoff, Am. J. Phys. J. Math. Chem. 39, 954 (1971).

60. V. Flügge, Practical Quantum Mechanics I, Springer, Berlin (1974).

61. R.L. Gibbs, Am. J. Phys. 43, 25 (1975).

62. J.J. Sakurai, Modern Quantum Mechanics, Menlo Park, Benjamin/Cummings (1985).

63. S. Bäßler, J. Phys. G-Nuclear and Particle Physics 36, 104005 (2009).

64. V.V. Nesvizhevsky, Usp. Fiz. Nauk, in print (2010).

65. V.V. Nesvizhevsky, H.G. Börner, A.M. Gagarski, G.A. Petrov, A.K. Petoukhov, H. Abele, S. Bäßler, Th. Stöferle, and S.M. Soloviev, Nucl. Instrum. Methods A440, 754 (2000).

66. T. Bowles, Nature 415, 267 (2002).

67. B. Schwarzschild, Physics Today 55, 20 (2002).

68. J. Hansson, D. Olevik, C. Turk, and H. Wiklund, Phys. Rev. D68, 108701 (2003).
69. V.V. Nesvizhevsky, A.K. Petoukhov, H.G. Börner, K.V. Protasov, A.Yu. Voronin, A. Westphal, S. Baessler, H. Abele, and A.M. Gagarski, Phys. Rev. D68, 108702 (2003).

70. V.V. Nesvizhevsky, Usp. Fiz. Nauk 46, 93 (2003).

71. V.V. Nesvizhevsky, Usp. Fiz. Nauk 47, 515 (2004).

72. V.V. Nesvizhevsky and K.V. Protasov, Quantum States of Neutrons in the Earth's Gravitational Field: State of the Art, Applications, Perspectives. in Edited book on Trends in quantum gravity research. New York, Nova science publishers (2006).

73. H. Abele, S. Bäßler, H.G. Börner, A.M. Gagarski, V.V. Nesvizhevsky, A.K. Petoukhov, K.V. Protasov, A.Yu. Voronin, and A. Westphal, AIP Conference Proceedings 842, 793 (2006).

74. A.Yu. Voronin, H. Abele, S. Bäßler, V.V. Nesvizhevsky, A.K. Petoukhov, K.V. Protasov, and A. Westphal, Phys. Rev. D73, 044029 (2006).

75. A.E. Meyerovich and V.V. Nesvizhevsky, Phys. Rev. A73, 063616 (2006).

76. R. Adhikari, Y. Cheng, A.E. Meyerovich, and V.V. Nesvizhevsky, Phys. Rev. A75, (2007) (2007).

77. A. Westphal, H. Abele, S. Bäßler, V.V. Nesvizhevsky, K.V. Protasov, and A.Yu. Voronin, Europ. Phys. J. C51, 367 (2007).

78. R. Cubitt, V.V. Nesvizhevsky, A.K. Petukhov, T. Soldner, A.Yu. Voronin, G. Pignol, K.V. Protasov, and P.V. Gurzhiyants, Nucl. Instrum. Methods A611, 322 (2009).

79. M. Kreuz, V.V. Nesvizhevsky, P. Schmidt-Wellenburg, T. Soldner, M. Thomas, H.G. Boerner, F. Naraghi, G. Pignol, K.V. Protasov, D. Rebreyend, F. Vezzu, R. Flaminio, C. Michel, L. Pinard, A. Remillieux, S. Bäßler, A.M. Gagarski, L.A. Grigorieva, T.M. Kuzmina, A.E. Meyerovich, L.P. Mezhov-Deglin, G.A. Petrov, A.V. Strelkov, and A.Yu. Voronin, Nucl. Instrum. Methods A611, 326 (2009).

80. V.V. Nesvizhevsky, Nucl. Instrum. Methods A557, 576 (2006).

81. V.V. Nesvizhevsky, G. Pignol, K.V. Protasov, G. Quemener, D. Forest, P. Ganau, J.M. Mackowski, Ch. Michel, J.L. Montorio, N. Morgado, L. Pinard, and A. Remillieux, Nucl. Instrum. Methods A578, 435 (2007).

82. G. Pignol, K.V. Protasov, D. Rebreyend, F. Vezzu, V.V. Nesvizhevsky, A.K. Petukhov, H.G. Börner, T. Soldner, P. Schmidt-Wellenburg, M. Kreuz, D. Forest, P. Ganau, J.M. Mackowski, C. Michel, J.L. Montorio, N. Morgado, L. Pinard, A. Remillieux, A.M. Gagarski, G.A. Petrov, A.M. Kusmina, A.V. Strelkov, H. Abele, S. Baeßler, and A.Yu. Voronin, GRANIT Project: a Trap for Gravitational Quantum States of UCN, arXiv:0708.2541. (2007).

83. P. Schmidt-Wellenburg, J. Barnard, P. Geltenbort, V.V. Nesvizhevsky, Ch. Plonka, T. Soldner, and O. Zimmer, Nucl. Instrum. Methods A577, 623 (2007).

84. J. Barnard and V.V. Nesvizhevsky, Nucl. Instrum. Methods A591, 431 (2008).

85. V.A. Artemiev, Atom. Energy 101, 901 (2006).

86. P.J. de Carli and J.C. Jameieson, Science 133, 18212 (1961).

87. A.E. Aleksenskii, M.V. Baidakova, A.Y. Vul, and V.I. Siklitskii, Phys. Solid State 41, 668 (1999). 
88. V.P. Alfimenkov, A.V. Strelkov, V.N. Shvetsov, V.V. Nesvizhevsky, A.P. Serebrov, R.R. Taldaev, and A.G. Kharitonov, JETP Lett. 55, 84 (1992).

89. V.V. Nesvizhevsky, A.V. Strelkov, P. Geltenbort, and P.S. Iaydjiev, Europ. J. Appl. Phys. 6, 151 (1999).

90. V.V. Nesvizhevsky, A.V. Strelkov, P. Geltenbort, and P.S. Iaydjiev, Phys. At. Nucl. 62, 776 (1999).

91. L. Bondarenko, E. Korobkina, V. Morozov, Yu. Panin, P. Geltenbort, and A. Steyerl, JETP Lett. 68, 691 (1998).

92. E.V. Lychagin, A.Yu. Muzychka, V.V. Nesvizhevsky, G.V. Nekhaev, R.R. Taldaev, and A.V. Strelkov, Phys. At. Nucl. 63, 548 (2000).

93. A.V. Strelkov, D.G. Kartashov, E.V. Lychagin, A.Yu. Muzychka, V.N. Shvetsov,V.V. Nesvizhevsky, P. Geltenbort, A.G. Kharitonov, A.P. Serebrov, R.R. Taldaev, J.M. Pendlebury, K. Schreckenbach, and P. Yaidjiev, Nucl. Instrum. Methods A440, 695 (2000).

94. V.V. Nesvizhevsky, E.V. Lychagin, A.Yu. Muzychka, G.V. Nekhaev, and A.V. Strelkov, Phys. Lett. B479, 353 (2000).

95. L.N. Bondarenko, P. Geltenbort, E.V. Korobkina, V.I. Morozov, and Yu.N. Panin, Phys. At. Nucl. 65, 11 (2002).

96. E.V. Lychagin, D.G. Kartashov, A.Yu. Muzychka, V.V. Nesvizhevsky, G.V. Nekhaev, and A.V. Strelkov, Phys. At. Nucl. 65, 19968 (2002).

97. A. Steyerl, G. Yerozolim, A.P. Serebrov, P. Geltenbort, N. Achiwa, Yu.N. Pokotilovski, O. Kwon, M.S. Lassakov, I.A. Krasnoschekova, and A.V. Vasilyev, Europ. Phys. J. B28, 299 (2002).
98. A.P. Serebrov, J. Butterworth, M. Daum, A.K. Fomin, P. Geltenbort, K. Kirch, I.A. Krasnoschekova, M.S. Lasakov, Yu.P. Rudnev, and V.E. Varlamov, Phys. Lett. A309, 218 (2003).

99. L.P. Mezhov-Deglin and A.M. Kokotin, JETP Lett. 70, 756 (1999).

100. S.I. Kisilev, V.V. Khmelenko, and D.M. Lee, J. Low Temp. Phys. 121, 671 (2000).

101. E.P. Bernard, R.E. Boltnev, V.V. Khmelenko, V. Kiryukhin, S.I. Kiselev, and D.M. Lee, Phys. Rev. B69, 104201 (2004).

102. L.P. Mezhov-Deglin, V.B. Efimov, A.A. Levchenko, G.V. Kolmakov, A.V. Lokhov, and V.V. Nesvizhevsky, AIP Conference Proc. (2006), vol. 850, p. 380.

103. L.P. Mezhov-Deglin, V.B. Efimov, A.V. Lokhov, E.V. Lychagin, A.Yu. Muzychka, V.V. Nesvizhevsky, and A.V. Strelkov, J. Low Temp. Phys. 148, 833 (2007).

104. V.V. Khmelenko, H. Kuntuu, and D.M. Lee, J. Low Temp. Phys. 148, 1 (2007).

105. L.P. Mezhov-Deglin, V.B. Efimov, A.V. Lokhov, A.A. Levchenko, G.V. Kolmakov, L.V. Abdurakhimov, M.Y. Brazhnikov, E.V. Lebedeva, R. May, V.V. Nesvizhevsky, A.Yu. Muzychka, E.V. Lychagin, and A.V. Strelkov, J. Low Temp. Phys. 150, 206 (2008).

106. V.V. Nesvizhevsky, G. Pignol, and K.V. Protasov, Low Temp. Phys. 850, 16792 (2006).

107. V.V. Nesvizhevsky, G. Pignol, and K.V. Protasov, Int. J. Nanoscience 6, 485 (2007). 